\title{
Critical Machine Cluster Identification using the Equal Area Criterion
}

\author{
Tilman Weckesser Hjörtur Jóhannsson Jacob Østergaard
}

\begin{abstract}
The paper introduces a new method to early identify the critical machine cluster (CMC) after a transient disturbance. For transient stability assessment with methods based on the equal area criterion it is necessary to split the generators into a group of critical and non-critical machines. The generators in the $\mathrm{CMC}$ are those likely to lose synchronism. The early and reliable identification of the CMC is crucial and one of the major challenges. The proposed new approach is based on the assessment of the rotor dynamics between two machines and the evaluation of their coupling strength. A novel coupling coefficient is derived and a cluster identification algorithm is developed. The algorithm determines the CMC based on the impact of the fault on the derived coupling coefficient of individual generator pairs. The results from two cases are presented and discussed, where the CMC is successfully determined just after fault clearance.
\end{abstract}

\section{INTRODUCTION}

$\mathbf{S}$ TABLE and secure supply of electric energy is a fundamental requirement of modern society. In order to ensure operation of the power system within stability and security limits, the system operator is dependent on tools and methods, which provide situational awareness. Analysis that determine if a system can remain within the stability \& security boundaries for a given set of credible contingencies are referred to as dynamic security assessment (DSA) [1]. An efficient transient stability assessment (TSA) method is an important part of a DSA toolbox. In order to reach real-time performance in TSA, approaches using so-called direct methods are appealing. A large number of these methods are either using the transient energy function, such as the BCU method [2], or the equal area criterion (EAC), such as the EEAC method [3] and the SIME method [4]. The mentioned methods employing the EAC are requiring that the generators in the system are split into a group of critical generators also called critical machine cluster (CMC), which is the group likely to lose synchronism, and a group of non-critical generators. This stems from the assumption that no matter how complex a system is the transient stability problem arises from the separation of two generator groups [4]. An early and reliable identification of the CMC is crucial to enable correct stability assessment.

In [4] it is proposed to extrapolate the individual rotor angle trajectories using a set of measurements and e.g. Taylor series expansion. The extrapolations are used to predict the individual rotor angles some time ahead. The predicted rotor angles are sorted descending and the gaps between entries are assessed. The generators are split into the critical and the non-critical group according to the maximum angular gap. [5] proposes

The authors are with the Centre for Electric Power and Energy, Department of Electrical Engineering, Technical University of Denmark, 2800 Lyngby, Denmark. (email: jtgw@elektro.dtu.dk)

Research supported by the Danish Council for Strategic Research (DSF).

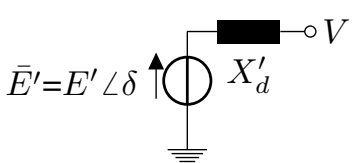

(a) Thevenin Equivalent

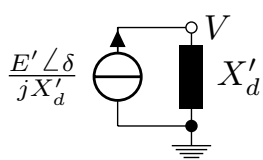

(b) Norton Equivalent
Fig. 1. Thévenin and Norton equivalents of generator an index for grouping the generators, which is called angle increment. It is a measure of the relative rotor angle change of an individual generator since fault clearance and with respect to the average change of the rotor angles of all generators.

In this paper a new method is proposed to identify the CMC, which utilizes efficient algorithms from graph theory. For that purpose, a novel coupling coefficient is derived, which represents the coupling strength between pairs of generators. The rest of the paper is structured as follows. In Sec. II the model used for the computation of the clustering coefficient as well as the EAC are briefly described. This is followed by the derivation of the one-machine equivalent (OME) of pairs of machines and the coupling strength coefficient. In Sec. III the cluster identification algorithm is introduced. Afterwards, in Sec. IV the results from test cases are presented and discussed. Finally, in Sec. V some concluding remarks are offered.

\section{Derivation of Coupling CoefFicients}

\section{A. Modelling for coupling analysis}

In order to derive the coupling coefficients, the synchronous generators are represented by the so-called "classical" transient stability model [6]. Each generator is represented by its Thévenin equivalent and correspondingly by an e.m.f. $E^{\prime}$ of constant magnitude behind the transient reactance $X_{d}^{\prime}$ (see Fig. 1a). This simple generator model is only valid for a very short time period, due to e.g. fast automatic voltage regulators. Therefore, the model is fitted to the current operating point using the latest measurements or simulation data. When the complex voltage $\bar{V}$ and the complex current injection $\bar{I}$ at the machine terminal are known, the e.m.f. can be computed.

$$
\bar{E}^{\prime}=E^{\prime} \angle \delta=\bar{V}+j X_{d}^{\prime} \bar{I}
$$

where $\delta$ corresponds to the rotor angle of the machine. When each generator is represented by its Norton equivalent (see Fig. 1b) and, hence, by a current source $\bar{E}^{\prime} / j X_{d}^{\prime}$ in parallel with the admittance corresponding to $X_{d}^{\prime}$, then the algebraic network equations describe the relation between the bus voltages, current injections and network admittances.

$$
\overline{\boldsymbol{I}}=\boldsymbol{Y} \overline{\boldsymbol{V}}
$$

Here, $\overline{\boldsymbol{I}}$ corresponds to the vector of the complex current injections at the generator buses, $\overline{\boldsymbol{V}}$ to the vector of complex 
bus voltages and $\boldsymbol{Y}$ to the "augmented" admittance matrix, which is obtained by adding the admittances corresponding to the transient reactances of the generators and loads to the original admittance matrix.

In order to compute the power injection of a generator as a function of the e.m.f.s of the remaining generators, the augmented admittance matrix $\boldsymbol{Y}$ needs to be extended to the internal nodes of the generators, which correspond to fictive nodes behind the respective $X_{d}^{\prime}$. Then the resulting extended admittance matrix $\boldsymbol{Y}_{\text {ex }}$ will be reduced to the internal nodes.

$$
\boldsymbol{Y}_{e x}=\left(\begin{array}{cc}
\boldsymbol{Y}^{T} & \boldsymbol{Y}_{b g} \\
\boldsymbol{Y}_{b g}^{T} & \boldsymbol{Y}_{g g}
\end{array}\right)
$$

$\boldsymbol{Y}_{b g}$ corresponds to the matrix representing the connection of the machine internal nodes to the respective machine terminal buses and $\boldsymbol{Y}_{b g}^{T}$ is its transpose. $\boldsymbol{Y}_{g g}$ corresponds to the square admittance matrix of order $m$, where $m$ is the number of generators and which holds the admittances to the corresponding $X_{d}^{\prime}$ on its diagonal. This extended and augmented admittance matrix allows computing the current injections $\overline{\boldsymbol{I}}_{\boldsymbol{t} \boldsymbol{r}}$ into the internal nodes as follows.

$$
\left(\begin{array}{c}
\mathbf{0} \\
\overline{\boldsymbol{I}}_{t r}
\end{array}\right)=\left(\begin{array}{cc}
\boldsymbol{Y} & \boldsymbol{Y}_{b g} \\
\boldsymbol{Y}_{b g}^{T} & \boldsymbol{Y}_{g g}
\end{array}\right)\left(\begin{array}{c}
\overline{\boldsymbol{V}} \\
\overline{\boldsymbol{E}}^{\prime}
\end{array}\right)
$$

where $\overline{\boldsymbol{E}}^{\prime}$ corresponds to a vector of the e.m.f.s. Here, the generators are again represented by their Thévenin equivalent.

In order to reduce the matrix to the internal nodes and eliminate all nodes, where no current injection takes place, a Kron reduction is carried out, which results in a reduced matrix $\boldsymbol{Y}_{\text {red }}$ and the algebraic equations of the reduced network.

$$
\overline{\boldsymbol{I}}_{t r}=Y_{\text {red }} \overline{\boldsymbol{E}}^{\prime}
$$

With (5) the apparent power injection $\bar{S}$ into the internal node of each machine can be computed as:

$$
\overline{\boldsymbol{S}}=\overline{\boldsymbol{E}}^{\prime} \overline{\boldsymbol{I}}_{t r}^{*}
$$

where $\overline{\boldsymbol{I}}_{t r}^{*}$ represents the vector of the complex conjugate of the current injections into the internal nodes. Following, the active power injection $P_{e, k}$ of generator $k$ can then be computed as a function of the e.m.f.s of the remaining generators.

$$
P_{e, k}=\sum_{l=1}^{m} E_{k}^{\prime} E_{l}^{\prime} Y_{k, l} \cos \left(\delta_{k}-\delta_{l}-\theta_{k, l}\right)
$$

with $\theta_{k, l}$ and $Y_{k, l}$ being the angle and the magnitude of the admittance $\left[\boldsymbol{Y}_{r e d}\right]_{k, l}=Y_{k, l} \angle \theta_{k, l}$. Under consideration of the "classical" model, the dynamics of the $k$-th machine are governed by the swing equation [6].

$$
\begin{gathered}
\dot{\delta}_{k}=\omega_{k} \\
\dot{\omega}_{k}=\ddot{\delta}_{k}=\frac{\omega_{0}}{M_{k}}\left(P_{m, k}-P_{e, k}\right)
\end{gathered}
$$

where $\omega_{0}$ corresponds to the synchronous speed, $M_{k}$ is the inertia coefficient and $P_{m, k}$ is the mechanical power.

\section{B. Equal area criterion}

The equal area criterion (EAC) allows determining transient stability of a one-machine infinite bus system (OMIB) without carrying out time-domain simulation. Therefore, a number of simplifications and assumptions are considered. The synchronous machine is represented by its Thévenin equivalent and the mechanical power is assumed to be constant. Furthermore, the machine's damping is neglected and the loads are represented by constant impedances. Under these assumptions, for a OMIB (9) can be rearranged as follows [6]:

$$
\left[\frac{d \delta}{d t}\right]^{2}=\int \frac{2}{M}\left(P_{m}-P_{e}(\delta)\right) d \delta
$$

If a function for the active electric power $P_{e}(\delta)$ is known, a stability margin can be computed as shown below.

$$
\eta=-\int_{\delta_{c}}^{\delta_{u}}\left[P_{m}-P_{e, P}(\delta)\right] d \delta-\int_{\delta_{0}}^{\delta_{c}}\left[P_{m}-P_{e, D}(\delta)\right] d \delta
$$

The subscripts $D$ and $P$ denote conditions during and after fault clearance respectively. $\delta_{c}$ is the rotor angle at fault clearance and $\delta_{u}$ the angle at the unstable equilibrium point. A positive margin represents a stable case, a negative an unstable.

\section{Formulation of a one-machine equivalent (OME)}

The proposed aggregation is similar to the one used in [7], where a OMIB equivalent of two aggregated groups of machines is computed. However, here the aim was to assess the dynamics of pairs of machines. For that purpose, a relative rotor angle $\phi_{i j}$ for each pair is determined as the difference of the rotor angles of generator $i$ and $j$.

$$
\phi_{i j}=\delta_{i}-\delta_{j}
$$

Similarly, the relative speed $\omega_{i j}$ and acceleration $\ddot{\phi}_{i j}$ can be computed.

$$
\begin{gathered}
\dot{\phi}_{i j}=\omega_{i j}=\omega_{i}-\omega_{j}=\dot{\delta}_{i}-\dot{\delta}_{j} \\
\ddot{\phi}_{i j}=\dot{\omega}_{i}-\dot{\omega}_{j}=\ddot{\delta}_{i}-\ddot{\delta}_{j}
\end{gathered}
$$

With (9) the relative acceleration may be rewritten as follows:

$$
\ddot{\phi}_{i j}=\frac{\omega_{0}}{M_{i}}\left(P_{m, i}-P_{e, i}\right)-\frac{\omega_{0}}{M_{j}}\left(P_{m, j}-P_{e, j}\right)
$$

As in [7] the inertia coefficient of the OME is computed as:

$$
M_{i j}=M_{i} M_{j} / M_{T} \text { with } M_{T}=M_{i}+M_{j}
$$

Following, the dynamics of the OME of a pair of machines can be described by a new swing equation.

$$
\ddot{\phi}_{i j}=\frac{\omega_{0}}{M_{i j}}\left(P_{m, i j}-P_{e, i j}\right)
$$

where

$$
\begin{aligned}
P_{m, i j} & =M_{T}^{-1}\left(M_{j} P_{m, i}-M_{i} P_{m, j}\right) \\
P_{e, i j} & =M_{T}^{-1}\left(M_{j} P_{e, i}-M_{i} P_{e, j}\right)
\end{aligned}
$$

Under the assumption that the mechanical power of both generators remains constant, the relative acceleration of the OME is dependent on the electric power of the individual machines. It can be shown that (18) can be reformulated as:

$$
P_{e, i j}\left(\phi_{i j}\right)=P_{c, i j}+P_{\max , i j} \sin \left(\phi_{i j}-\nu_{i j}\right)
$$


where

$$
\begin{gathered}
P_{c, i j}=\frac{1}{M_{T}}\left[M_{j} E_{i}^{\prime 2} Y_{i i} \cos \left(\theta_{i i}\right)-M_{i} E_{j}^{\prime 2} Y_{j j} \cos \left(\theta_{j j}\right)\right] \\
P_{\max , i j}=\sqrt{D_{i j}^{2}+C_{i j}^{2}} \text { and } \nu_{i j}=-\tan ^{-1} \frac{C_{i j}}{D_{i j}}
\end{gathered}
$$

with

$$
\begin{aligned}
C_{i j}= & \frac{1}{M_{T}}\left[M_{j} \sum_{k=1}^{m} E_{i}^{\prime} E_{k}^{\prime} Y_{i k} \cos \left(\delta_{j}-\delta_{k}-\theta_{i k}\right)\right. \\
& \left.-M_{i} \sum_{k=1}^{m} E_{j}^{\prime} E_{k}^{\prime} Y_{j k} \cos \left(\delta_{i}-\delta_{k}-\theta_{j k}\right)\right] \\
D_{i j}= & -\frac{1}{M_{T}}\left[M_{j} \sum_{k=1}^{m} E_{i}^{\prime} E_{k}^{\prime} Y_{i k} \sin \left(\delta_{j}-\delta_{k}-\theta_{i k}\right)\right. \\
& \left.+M_{i} \sum_{k=1}^{m} E_{j}^{\prime} E_{k}^{\prime} Y_{j k} \sin \left(\delta_{i}-\delta_{k}-\theta_{j k}\right)\right]
\end{aligned}
$$

With (19) the swing equation of the OME can be expressed as follows, which allows computation of the EAC.

$$
\ddot{\phi}_{i j}=\frac{\omega_{0}}{M_{i j}}\left[P_{m, i j}-\left(P_{c, i j}+P_{\max , i j} \sin \left(\phi_{i j}-\nu_{i j}\right)\right)\right]
$$

\section{Coupling coefficients}

In this analysis the coupling strength between two generators is defined as the available excess dissipation energy and, hence, it can be computed as the difference between available dissipation energy after fault clearance and kinetic energy gained during the fault. In order to determine the coupling coefficient, (10) can be reformulated as follows.

$$
\frac{1}{2} \frac{M_{i j}}{\omega_{0}} \dot{\phi}_{i j}^{2}=\int P_{a, i j} d \phi_{i j}
$$

where $\dot{\phi}_{i j}=\omega_{i j}$ and $P_{a, i j}$ with (17) \& (19) is described as:

$$
P_{a, i j}=P_{m, i j}-\left[P_{c, i j}+P_{\max , i j} \sin \left(\phi_{i j}-\nu_{i j}\right)\right]
$$

Under consideration of the EAC, in (23) the left-side term represents the kinetic energy $E_{k i n, i j}$ and the right-side the dissipation energy $E_{d i s, i j}$, which corresponds to the maximum kinetic energy that the OME can absorb. The OME is stable, if the available $E_{d i s, i j}$ is greater or equal to $E_{k i n, i j}$ induced by the fault. Therefore, it is proposed that the coupling strength is approximated by the coupling coefficient $c_{i j}$.

$$
c_{i j}=E_{d i s, i j}-E_{k i n, i j}
$$

where

$$
\begin{aligned}
& E_{d i s, i j}=-\int_{\phi_{c, i j}}^{\phi_{u, i j}} P_{m, i j}-P_{e, i j}\left(\phi_{i j}\right) d \phi_{i j} \\
& E_{k i n, i j}=\frac{1}{2} \frac{M_{i j}}{\omega_{0}} \omega_{i j}^{2}
\end{aligned}
$$

The proposed coupling strength $c_{i j}$ is negative, when the coupling is very weak and the two machines are likely to lose synchronism, and it is positive, when there is strong coupling, which makes it likely that the two machines remain in synchronism. By computing the coupling strength between each pair of machines a coupling matrix $\mathbf{H}$ can be constructed.

\section{E. Impact of fault on coupling strength}

The coupling matrix $\mathbf{H}$ can be computed for steady state and dynamic system conditions. In steady state the relative speed between the generators is equal to zero and, hence, the coupling coefficients are solely determined by the available dissipation energy. In order to determine the CMC after the occurrence and the clearance of a fault, the change in coupling strength due to the fault was found to be essential. Therefore,

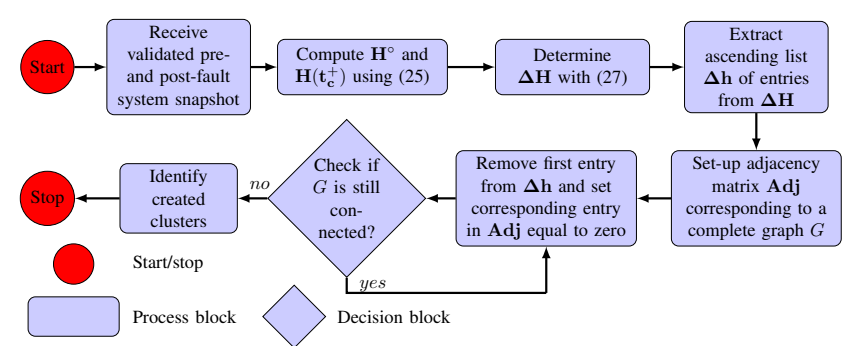

Fig. 2. Block diagram of the critical cluster identification algorithm.

it is proposed to use $\boldsymbol{\Delta} \mathbf{H}$, which is the difference in coupling strength between post- and pre-fault condition.

$$
\Delta \mathbf{H}=\mathbf{H}\left(t_{c}^{+}\right)-\mathbf{H}^{\circ}
$$

where $\mathbf{H}\left(t_{c}^{+}\right)$is the coupling matrix determined at the time just after fault clearance $t_{c}^{+}$and $\mathbf{H}^{\circ}$ is the coupling matrix determined at steady state before the fault. By using the difference in coupling strength, focus is set on changes, structural as well as dynamic, due to the fault. If the coupling between two generators is weakened in the post-fault condition the corresponding entry in $\mathbf{\Delta H}$ becomes negative, while a strengthening of the coupling results in a positive value.

\section{CRitical Machine Cluster Identification}

In order to identify the CMC, an algorithm was developed. A block diagram of the algorithm is shown in Fig. 2. In this approach two snapshots, one of the pre- and one of the post-fault system condition, are used to compute the coupling matrices $\mathbf{H}^{\circ}$ and $\mathbf{H}\left(t_{c}^{+}\right)$. These matrices are then used to compute the change in coupling strength between individual generators due to the fault, which results in the matrix $\Delta \mathbf{H}$. The entries of $\Delta \mathbf{H}$ are copied into the vector $\Delta \mathbf{h}$, where the entries are sorted ascending. Moreover, an adjacency matrix $A d j$ is initialized with the same dimension as $\mathbf{\Delta H}$ and corresponding to a complete graph $G$, where each node of $G$ corresponds to a generator. In order to identify the $\mathrm{CMC}$, individual edges are removed one by one from $G$ until the graph is split. The edges are removed beginning with the lowest change in coupling strength and according to the list of increasing changes in $\Delta \mathbf{h}$. After each removal a depth-first search (DFS) is carried out to check if $G$ is still connected. If a splitting of the graph occurred, a second DFS based algorithm is executed to identify the created clusters. Since each node of $G$ corresponds to a generator in the system, a splitting of the graph corresponds to a separation of the generators into clusters and the proposed approach is designed to identify the weakest coupled clusters.

\section{RESUlTS FOR CMC IDENTIFICATION}

\section{A. Test system and scenario}

The power system model, employed to test the presented method, is the New England \& New York system described in [8]. It consists of 68 buses and 16 generators. The loads are modelled as constant impedances and the generators are represented by a sixth order model in the time-domain simulation. All generators have a simple excitation and voltage regulation system as well as a thermal turbine/governor model. Moreover, 


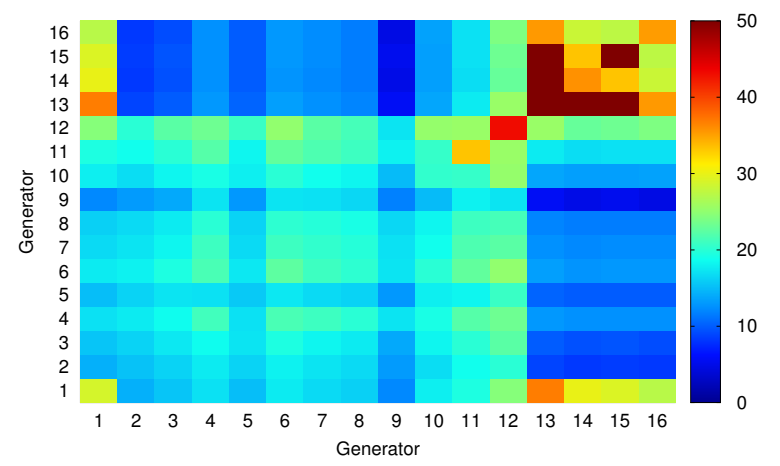

Fig. 3. Coupling matrix computed by (25) in steady state initial conditions.

all generators, but GEN 7 and GEN 14, are equipped with a power system stabilizer. The initial state of the system was as well adopted from [8]. To test the proposed method three-phase short circuits on individual transmission lines were considered, which last for $250 \mathrm{~ms}$ and are cleared by opening of the breakers at both ends of the respective transmission line.

\section{B. Coupling strength at initial conditions}

In the following the coupling strength matrix is computed for the pre-fault steady state conditions with the equations derived in Sec. II-D, the results are used to show the apparent correlation of the proposed coupling coefficients and the structure of the power system. The coupling matrix in the initial conditions is shown in Fig. 3. The obtained coupling coefficients of the generators are compared to each other relatively, where greater values, e.g. above 25 , indicate a stronger coupling, while lower values, e.g. below 20 , correspond to a weaker coupling. Visual inspection of the graph allows identifying patterns, which may indicate a stronger coupling between certain generators. The coupling coefficients between the generators GEN $1 \& 13-16$ may suggest a tight coupling. In the one-line diagram, which can be found in e.g. [9], it can be seen that the generators GEN $14-16$ are close and well connected. Moreover, generator GEN 1 is strongly connected with two transmission lines to the area, which may explain the strong coupling to this generator group. The generators GEN $4-8 \& 10-11$ seem to be tightly coupled as well. The coupling strength of generator GEN 12 appears to be relatively high with respect to all generators but generator GEN 9. This could indicate that generator GEN 12 has a large impact on all generators, which may also be explained by its central location in the system. GEN 9 appears to have weaker coupling to the other generators. This may be explained by its remote location and the few lines connecting it to the rest of the system.

\section{Case 1: Transient stable}

In this section an example case is shown, where a transient disturbance occurs but does not lead to a loss of synchronism of generators. The considered contingency is a three-phase short circuit on the transmission line connecting bus 1 and 30 .

1) Rotor angle response: The rotor angle response of a selection of generators is shown in Fig. 4. The selection contains the two generators GEN $10 \& 11$, which are strongest effected by the fault, six generators (GEN 1, $4 \& 6-9$ ),

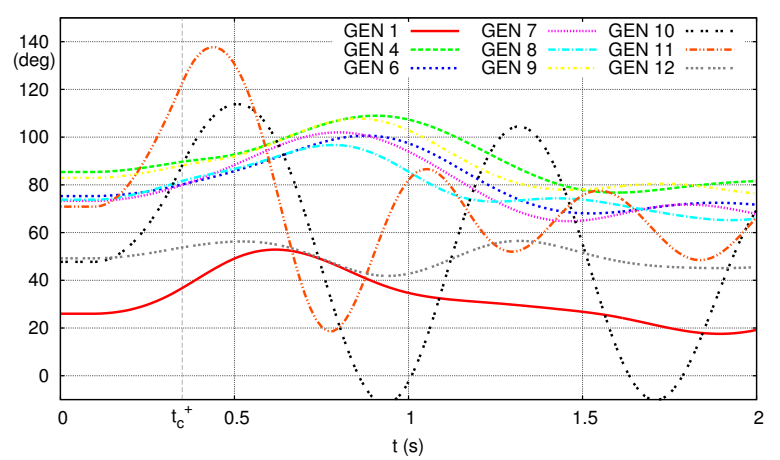

Fig. 4. Case 1: Rotor angle response of a selection of generators.

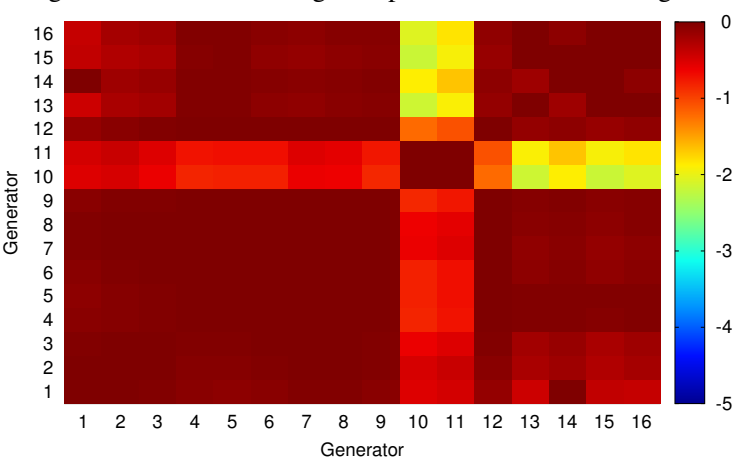

Fig. 5. Case 1: $\boldsymbol{\Delta} \mathbf{H}$ computed by (27) at $t_{c}^{+}$.

which experience a less significant deviation in rotor angle, and generator GEN 12, which is in the vicinity of the fault but barely effected. Although the fault causes significant oscillation of the rotor angles of generator GEN $10 \& 11$, the system remains stable and returns to a new steady state.

2) Critical cluster identification: The matrix $\Delta \mathbf{H}$ can be displayed as a two-dimensional grid, where the matrix entries and, consequently, the changes in coupling strength are displayed by different colors. Figure 5 shows the changes in coupling strength computed at $t_{c}^{+}$. The graph suggests that the coupling strength between most generators was barely affected by the fault. However, the coupling of GEN $10 \& 11$ to the remaining generators appears to be weakened by the fault, which is well aligned with the observations from Fig. 4.

When the proposed algorithm is employed, individual connections of the graph corresponding to the values in $\mathbf{\Delta H}$ are removed beginning with the largest negative change. The removing of the connections is done by setting the corresponding entries in the adjacency matrix to zero. Figure 6 shows the adjacency matrix at the instance when the proposed clustering algorithm splits the graph into two. The proposed method identifies by employing the DFS based algorithm presented in Fig. 2 that GEN $10 \& 11$ are the generators with the weakest coupling to the remaining system. This is well aligned with the observations described in Sec. IV-C1.

\section{Case 2: Transient unstable}

In this section the performance of the method is presented on a transient unstable case. The contingency is a three-phase short circuit on the transmission line connecting bus 4 and 5 .

1) Rotor angle response: The rotor angle responses of a selection of generators are shown in Fig. 7. The selection 


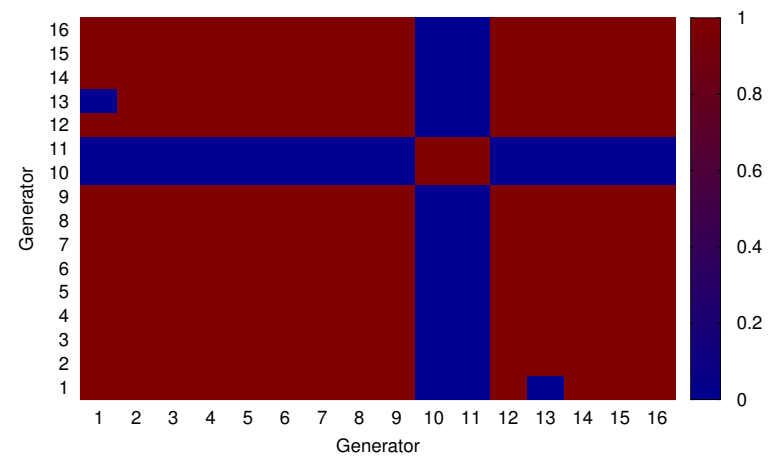

Fig. 6. Case 1: Adjacency matrix after graph split into two disjoint subsets.

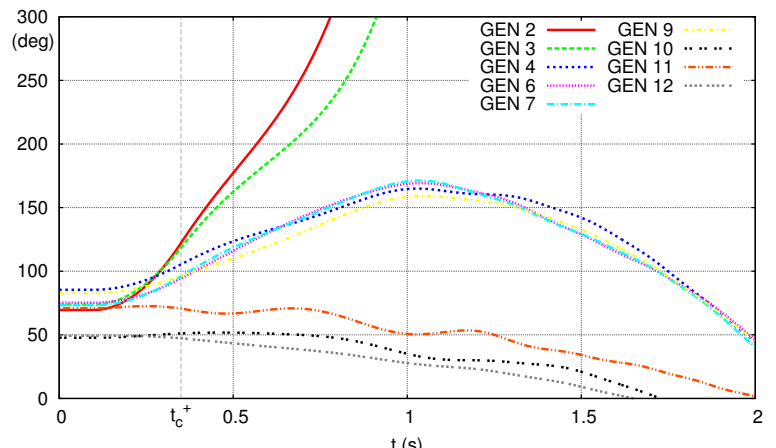

Fig. 7. Case 2: Rotor angle response of a selection of generators.

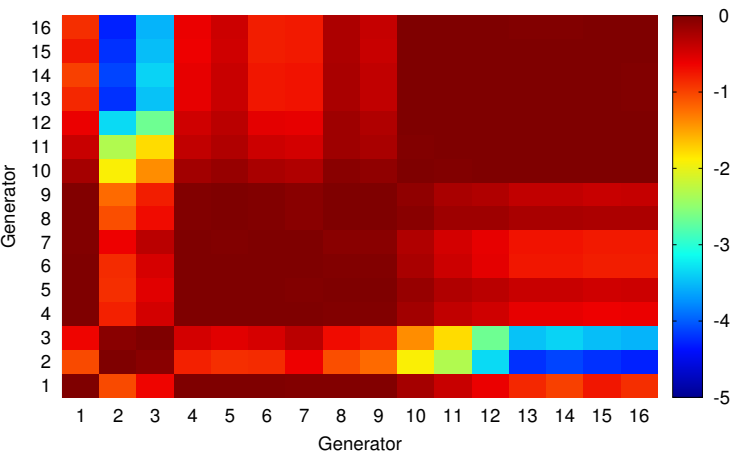

Fig. 8. Case 2: $\boldsymbol{\Delta} \mathbf{H}$ computed by (27) at $t_{c}^{+}$.

contains the two generators GEN 2 \& 3, which are losing synchronism, the generators GEN 4, 6, $7 \& 9$, which are also significantly impacted by the fault, and GEN $10-12$, which are initially less affected by the fault. The loss of generator GEN $2 \& 3$ is leading to a considerable lag of power, which leads to a system collapse. The rotor angles at $t_{c}^{+}$may suggest that the generators initially splits into two clusters, where the first consists of GEN $2-4,6,7 \& 9$ and the second of GEN $10-12$. Later the generators split into three groups namely, GEN $2 \& 3$, GEN 4, 6, $7 \& 9$ and GEN $10-12$.

2) Critical cluster identification: Figure 8 shows the change in coupling strength. The visual inspection of the graph allows identifying three machine clusters. The first cluster is GEN $1 \& 4-9$, the second cluster is GEN $10-16$ and the third cluster is GEN $2 \& 3$. This is in good agreement with the observations extracted from the rotor angles shown in Fig. 7.

The $\mathbf{\Delta H}$ matrix is again forwarded to the cluster identification algorithm, where the matrix is converted into a graph. Figure 9 shows the adjacency matrix of the corresponding

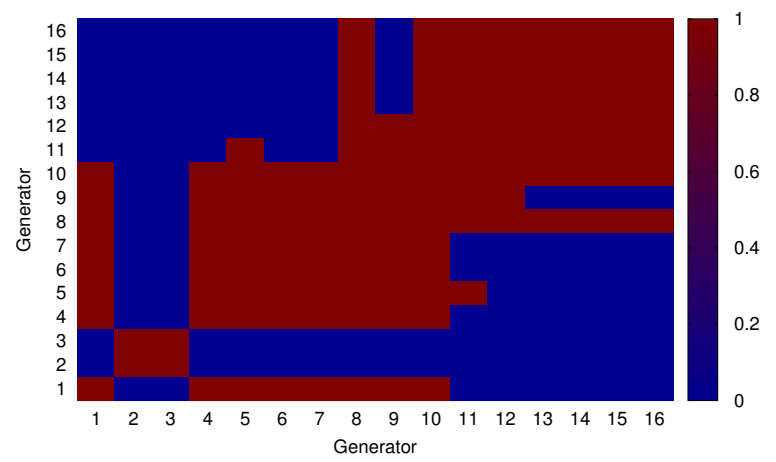

Fig. 9. Case 2: Adjacency matrix after graph split into two disjoint subsets. graph, when the first decoupled set of generators is identified with the proposed algorithm. It can be seen that all connections from generators GEN $2 \& 3$ are removed and, hence, already at $t_{c}^{+}$the algorithm identifies those generators as the CMC.

\section{CONCLUSION}

In this paper a new approach was proposed for identifying the $\mathrm{CMC}$ after a transient disturbance. For that purpose, a novel coupling coefficient was derived, which is determining the coupling strength of each pair of generators. This coefficient can be used to set up a coupling matrix, which describes the coupling strength between all generators in the system. In order to determine the $\mathrm{CMC}$ after a fault, the coupling strength matrix is determined for the initial condition and just after fault clearance. The difference of the two matrices is a new matrix, which describes the change of the coupling strength due to the fault and can be utilized to identify the CMC. A method, which uses efficient algorithms from graph theory, was proposed to identify the CMC. In two test cases it was demonstrated that although the coupling coefficients do not encompass all the existing controls, the clustering algorithm allows early and correct determination of the CMC. Another application of the developed cluster coefficients may be the early identification of stable cases, which will be further investigated.

\section{REFERENCES}

[1] K. Morison, L. Wang, and P. Kundur, "Power system security assessment," IEEE Power and Energy Magazine, vol. 2, no. october, 2004.

[2] H.-D. Chiang, F. F. Wu, and P. P. Varaiya, "A BCU Method for Direct Analysis of Power System Transient Stability," IEEE Transactions on Power System, vol. 9, no. 3, pp. 1194-1208, Aug. 1994.

[3] Y. Xue, T. Van Cutsem, and M. Ribbens-Pavella, "Extended equal area criterion justifications, generalizations, application," IEEE Transaction on Power Systems, vol. 4, no. 1, pp. 44-52, Feb. 1989.

[4] M. Pavella, D. Ernst, and D. Ruiz-Vega, Transient Stability of Power Systems: A Unified Approach to Assessment and Control. Kluwer Academic Publishers, 2000.

[5] J. Lee, B. Lee, S.-H. Kwon, H.-K. Nam, J.-B. Choo, and K. Yi, "Fast contingency screening for on-line transient stability monitoring of the KEPCO system," in 2001 PES Summer Meeting. Conference Proceedings, vol. 1. IEEE, 2001, pp. 314-319.

[6] P. Kundur, Power System Stability and Control, N. J. Balu and M. G. Lauby, Eds. McGraw-Hill Inc., 1994.

[7] Y. Xue and M. Pavella, "Extended equal-area criterion: an analytical ultra-fast method for transient stability assessment and preventive control of power systems," Int. J. Elec. Power, vol. 11, no. 2, Apr. 1989.

[8] G. Rogers, Power System Oscillations. Springer, 2000.

[9] D. Molina, J. Liang, R. G. Harley, and G. K. Venayagamoorthy, "Virtual Generators: Simplified Online Power System Representations for WideArea Damping Control,” in IEEE PES General Meeting, Jul. 2012. 